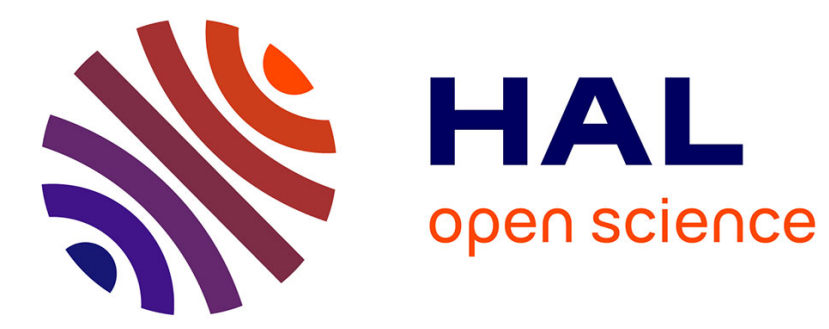

\title{
Interval Observer Design for Estimation and Control of Time-Delay Descriptor Systems
}

Denis Efimov, Andrey Polyakov, Jean-Pierre Richard

\section{To cite this version:}

Denis Efimov, Andrey Polyakov, Jean-Pierre Richard. Interval Observer Design for Estimation and Control of Time-Delay Descriptor Systems. European Journal of Control, 2015, 23 (5), pp.26-35. 10.1016/j.ejcon.2015.01.004 . hal-01108432

\section{HAL Id: hal-01108432 \\ https://hal.inria.fr/hal-01108432}

Submitted on 22 Jan 2015

HAL is a multi-disciplinary open access archive for the deposit and dissemination of scientific research documents, whether they are published or not. The documents may come from teaching and research institutions in France or abroad, or from public or private research centers.
L'archive ouverte pluridisciplinaire HAL, est destinée au dépôt et à la diffusion de documents scientifiques de niveau recherche, publiés ou non, émanant des établissements d'enseignement et de recherche français ou étrangers, des laboratoires publics ou privés. 


\title{
Interval Observer Design for Estimation and Control of Time-Delay Descriptor Systems
}

\author{
Denis Efimov, Andrey Polyakov, Jean-Pierre Richard
}

\begin{abstract}
The problem of interval observer design is addressed for a class of descriptor linear systems with time delays. First, an interval observation for any input in the system is provided. Second, the control input is designed together with the observer gains in order to guarantee interval estimation and stabilization simultaneously. Efficiency of the proposed approach is illustrated by numerical experiments with Leontief delayed model.
\end{abstract}

\section{Index Terms}

Interval Observers, Descriptor Systems, Time-delay Systems

\section{INTRODUCTION}

The state estimation problem for uncertain models of industrial plants or biological systems has a great practical importance [1], [2], [3]. The model uncertainty can be presented by unknown (possibly time-varying) parameters, external disturbances and/or measurement noises. In such a way the designed estimator has to ensure a certain robustness of generated estimates with respect to perturbations. Another issue is that the observer design is structurally complicated in this case, since all uncertain terms should be either estimated simultaneously or avoided in the observer equations (e.g. substituted by some known bounds).

In such a case an important characteristic appears dealing with accuracy of the generated estimates in the presence of all perturbations (unknown parameters, exogenous disturbances, measurement noise). The problem of accuracy evaluation is partially related with the problem of quantitative estimation of robustness. The difference is that usually for robustness quantification a gain should be computed between the maximal amplitude of perturbation and the maximal amplitude of response (the state estimation error in our case), while for accuracy evaluation the deviations from the nominal values have to be computed as tight as possible. It is strongly appreciated in applications to estimate this accuracy either off-line (during the design phase) or on-line using some numerical routines. The set-membership estimation algorithms dispose this important advantage [4], [5], [6].

There exist many approaches to design state observers for uncertain systems [1], [2], [3], all of them are heavily related with the type of the plant model. A special class of models is composed by the so-called (linear) continuous-time descriptor systems (singular systems or differential-algebraic systems) [7]. Descriptor systems have attracted much attention due to the numerous applications in economics (Leontief dynamic model) [8], in electrical engineering [9], mechanical systems with constraints [10] or flow optimal control [11]. Another important class of models is described by time-delay differential equations [12]. The problem of observer design for delayed systems

The authors are with the Non-A project @ Inria Lille, Parc Scientifique de la Haute Borne, 40 avenue Halley, 59650 Villeneuve d'Ascq, France and with LAGIS UMR 8219, Ecole Centrale de Lille, Avenue Paul Langevin, 59651 Villeneuve d'Ascq, France, \{denis.efimov; andrey.polyakov; jean-pierre.richard\}@inria.fr. The first author is with the Department of Control Systems and Informatics, University ITMO, 49 avenue Kronverkskiy, 197101 Saint Petersburg, Russia.

This work is supported in part by Region Nord-Pas de Calais, by the Government of Russian Federation (Grant 074-U01) and the Ministry of Education and Science of Russian Federation (Project 14.Z50.31.0031). 
is rather complex [13], as well as the stability conditions for analysis of functional differential equations are rather complicated [14], [15]. Especially the observer synthesis is problematical if the model of a delayed system contains parametric and signal uncertainties, or when the delay is time-varying or uncertain [16], [17], [18], [19], [20].

The present work deals with an intersection of these classes, i.e. with linear descriptor systems subject to a constant time delay. An observer solution for this more complex situation may be demanded in many real-world applications (economics, electrical circuits, flow control systems, and so on). Inclusion in a descriptor model of (almost always presented) delay effects increases accuracy of modeling (among others, in the case of convection effects in a fluid flow). In addition, a delayed descriptor system is a combination of two dynamics: a differential equation with a difference equation (in the delay-free case, algebraic constraint is static), which enlarges the class of physical phenomenon that can be modeled in this framework.

The proposed solution of estimation problem for these systems is based on the set-membership estimation approaches [4], [5], [6], and in particular a design of interval observers [21], [22], [4], [23], [24], [25], [26] is presented in this work. Some results have been obtained recently on synthesis of interval observers for timedelay systems [19], [20], and the objective is to extend these design tools to the class of descriptor systems. The advantages of the interval observers are that they are well adapted for observer design for highly uncertain systems (if the intervals of admissible values for unknown terms are given) and that they are capable to provide asymptotically rather tight bounds on the estimation accuracy, since the interval of admissible values for the state at each instant of time is evaluated.

The outline of this work is as follows. Some preliminary results are given in Section 2. Problem statement is presented in Section 3. Main results are formulated in Section 4. Numerical experiments and concluding remarks are presented in sections 5 and 6 respectively.

\section{PReliminaries}

In the rest of the paper, the following definitions will be used:

- $\mathbb{R}$ is the set of all real numbers $\left(\mathbb{R}_{+}=\{\tau \in \mathbb{R}: \tau \geq 0\}\right), \mathbb{C}$ is the set of complex numbers; $\mathcal{C}_{\tau}=C([-\tau, 0], \mathbb{R})$ is the set of continuous maps from $[-\tau, 0]$ into $\mathbb{R} ; \mathcal{C}_{\tau+}=\left\{y \in \mathcal{C}_{\tau}: y(s) \in \mathbb{R}_{+}, s \in[-\tau, 0]\right\}$;

- $x_{t}$ is an element of $\mathcal{C}_{\tau}^{n}$ associated with a map $x_{t}: \mathbb{R} \rightarrow \mathbb{R}^{n}$ by $x_{t}(s)=x(t+s)$, for all $s \in[-\tau, 0]$;

- $|x|$ denotes the absolute value of $x \in \mathbb{R},\|x\|$ is the Euclidean norm of a vector $x \in \mathbb{R}^{n},\|\varphi\|=$ $\sup _{t \in[-\tau, 0]}\|\varphi(t)\|$ for $\varphi \in \mathcal{C}_{\tau}^{n},\|A\|$ corresponds to the Euclidean induced norm for a matrix $A \in \mathbb{R}^{n \times n}$;

- for a measurable and locally essentially bounded input $u: \mathbb{R}_{+} \rightarrow \mathbb{R}^{p}$ the symbol $\|u\|_{\left[t_{0}, t_{1}\right]}$ denotes its $L_{\infty}$ norm $\|u\|_{\left[t_{0}, t_{1}\right]}=e s s \sup \left\{\|u(t)\|, t \in\left[t_{0}, t_{1}\right]\right\}$, the set of all such inputs $u$ with the property $\|u\|_{[0,+\infty)}<\infty$ will be denoted as $\mathcal{L}_{\infty}^{p}$;

- for a matrix $A \in \mathbb{R}^{n \times n}$ the vector of its eigenvalues is denoted as $\lambda(A)$;

- $1_{n} \in \mathbb{R}^{n}$ is stated for a vector with unit elements, $I_{n}$ denotes the identity matrix of dimension $n \times n$;

- $a \mathcal{R} b$ corresponds to an elementwise relation $\mathcal{R} \in\{<,>, \leq, \geq\}$ ( $a$ and $b$ are vectors or matrices): for example $a<b$ (vectors) means $\forall i: a_{i}<b_{i}$; for $\phi, \varphi \in \mathcal{C}_{\tau}^{n}$ the relation $\phi \mathcal{R} \varphi$ has to be understood elementwise for all domain of definition of the functions, i.e. $\phi(s) \mathcal{R} \varphi(s)$ for all $s \in[-\tau, 0]$;

- the relation $P \prec 0(P \succ 0)$ means that the matrix $P \in \mathbb{R}^{n \times n}$ is negative (positive) definite.

\section{A. Descriptor linear systems}

Consider a descriptor system

$$
E \dot{x}(t)=A_{0} x(t)+B u(t)
$$


where $x \in \mathbb{R}^{n}$ and $u(t) \in \mathcal{L}_{\infty}^{m}$ are the state and the input (the matrices $E \in \mathbb{R}^{n \times n}, A_{0} \in \mathbb{R}^{n \times n}$ and $B \in \mathbb{R}^{n \times m}$ ), which is called regular if the characteristic polynomial $\operatorname{det}\left(s E-A_{0}\right)$ does not vanish identically for all $s \in \mathbb{C}$ [27], [28]. In the regular case there exist matrices $P, Q \in \mathbb{R}^{n \times n}$ such that

$$
Q E P=\left[\begin{array}{cc}
I_{n_{1}} & 0 \\
0 & N
\end{array}\right], Q A_{0} P=\left[\begin{array}{cc}
J & 0 \\
0 & I_{n_{2}}
\end{array}\right],
$$

where $n_{2}=n-n_{1}$ for some $1 \leq n_{1}<n, N \in \mathbb{R}^{n_{2} \times n_{2}}$ and $J \in \mathbb{R}^{n_{1} \times n_{1}}$ are in the Jordan canonical form, the matrix $N$ is nilpotent of index $\nu$ (i.e. $N^{\nu}=0$ and $N^{\nu-1} \neq 0$ ). The descriptor system (or the pair $\left(E, A_{0}\right)$ ) has index that is the index of nilpotence $\nu$ of $N$. If a descriptor system has index $\nu>1$, then it admits impulsive solutions.

\section{B. Descriptor linear time-delay systems}

Consider a descriptor time-delay system

$$
E \dot{x}(t)=A_{0} x(t)+A_{1} x(t-\tau)+B u(t)
$$

where $\tau>0$ is the delay and $x(t) \in \mathbb{R}^{n}$ is the state, $x_{0} \in \mathcal{C}_{\tau}^{n}$ is the initial condition, $u(t) \in \mathcal{L}_{\infty}^{m}$. The system (1) has an index that is equal to the index of the pair $\left(E, A_{0}\right)$. If $\nu>1$ then the descriptor time-delay system has impulsive solutions. If a regular descriptor system has index 1 , then it can be presented in the following canonical form (similar case has been considered in [27]), where

$$
E=\left[\begin{array}{cc}
I_{n_{1}} & 0 \\
0 & 0
\end{array}\right] ; B=\left[\begin{array}{c}
B_{1} \\
B_{2}
\end{array}\right] ; A_{i}=\left[\begin{array}{cc}
A_{i 1} & A_{i 2} \\
A_{i 3} & A_{i 4}
\end{array}\right], i=0,1 .
$$

Denote in this case $x=\left[x_{1}^{\mathrm{T}} x_{2}^{\mathrm{T}}\right]^{\mathrm{T}}$, where $x_{1} \in \mathbb{R}^{n_{1}}$ and $x_{2} \in \mathbb{R}^{n_{2}}$. Note that this canonical representation is not unique, indeed for any nonsingular matrix $\Upsilon \in \mathbb{R}^{n_{2} \times n_{2}}$,

$$
E=\left[\begin{array}{cc}
I_{n_{1}} & 0 \\
0 & 0
\end{array}\right] ; B=\left[\begin{array}{c}
B_{1} \\
\Upsilon B_{2}
\end{array}\right] ; A_{i}=\left[\begin{array}{cc}
A_{i 1} & A_{i 2} \\
\Upsilon A_{i 3} & \Upsilon A_{i 4}
\end{array}\right], i=0,1
$$

is again a canonical representation of (1) in the same coordinates $x$. If it is assumed that $\operatorname{det}\left(A_{04}\right) \neq 0$ (that is the case considered below), then (2) can be selected in the following form for $\Upsilon=-A_{04}^{-1}$ :

$$
E=\left[\begin{array}{cc}
I_{n_{1}} & 0 \\
0 & 0
\end{array}\right] ; B=\left[\begin{array}{c}
B_{1} \\
B_{2}
\end{array}\right] ; A_{0}=\left[\begin{array}{cc}
A_{01} & A_{02} \\
A_{03} & -I_{n_{2}}
\end{array}\right], A_{1}=\left[\begin{array}{cc}
A_{11} & A_{12} \\
A_{13} & A_{14}
\end{array}\right] .
$$

Proposition 1. [27] Assume that $\operatorname{det}\left(A_{04}\right) \neq 0$ and $x_{0} \in \mathcal{C}_{\tau}^{n}$, then for any $u(t) \in \mathcal{L}_{\infty}^{m}$ an absolute continuous solution (see [9]) of (1) exists for all $t \in \mathbb{R}_{+}$and it is unique.

The notion of (global) asymptotic stability for (1) is understood in the standard for time-delay systems sense [12].

Proposition 2. [27] Assume that $\operatorname{det}\left(A_{04}\right) \neq 0$ and $\max _{1 \leq i \leq n_{2}}\left|\lambda_{i}\left(A_{04}^{-1} A_{14}\right)\right|<1$. If there exist $P \in \mathbb{R}^{n \times n}$,

$$
P=\left[\begin{array}{cc}
P_{1} & 0 \\
P_{2} & P_{3}
\end{array}\right], P_{1}=P_{1}^{T} \succ 0, P_{1} \in \mathbb{R}^{n_{1} \times n_{1}}, P_{3} \in \mathbb{R}^{n_{2} \times n_{2}},
$$


and $U \in \mathbb{R}^{n \times n}, U=U^{T} \succ 0$ that satisfy the following LMI for some $\gamma>0$ :

$$
\begin{aligned}
& {\left[\begin{array}{ccc}
\Psi & P^{T} B & P^{T} A_{1} \\
B^{T} P & -\gamma^{2} I_{m} & 0 \\
A_{1}^{T} P & 0 & -U
\end{array}\right] \prec 0,} \\
& \Psi=P^{T} A_{0}+A_{0}^{T} P+U+I_{n},
\end{aligned}
$$

then the system (1), (2) is globally asymptotically stable for $u \equiv 0$ and any delay $\tau>0$, in addition its $H_{\infty}$ gain from the input $u$ to the state $x$ is less than $\gamma$.

\section{Positive descriptor linear time-delay systems}

Positive (nonnegative) or cooperative (monotone) models [29], [30], [31], [32] have a large area of applications for description the systems of different nature in biology, economics, chemistry etc.

Definition 1. A regular descriptor system (1) with index 1 is called positive if the restrictions $x_{0} \in \mathcal{C}_{\tau+}^{n}, u(t) \in \mathbb{R}_{+}^{m}$ for all $t \geq 0$ imply that $x(t) \in \mathbb{R}_{+}^{n}$ for all $t \geq 0$.

The generic results on positivity of the descriptor linear systems (without time delays) and their stability results are presented in [28], [33]. In the regular case with index 1 they can be seriously simplified [33]. In the spirit of these results, for time delay systems the following extensions can be obtained. For this purpose recall that a matrix $A$ is called Metzler if all its off-diagonal elements are nonnegative, a matrix $A$ is called nonnegative if $A \geq 0$ (elementwise).

Proposition 3. For a regular descriptor system (1) with index 1, written in the canonical form (2) with $\operatorname{det}\left(A_{04}\right) \neq 0$, the following properties are equivalent:

(a) it is positive;

(b) there exists representation (2) such that $A_{04}$ is Hurwitz, $A_{0}$ is Metzler and $A_{1}, B$ are nonnegative;

(c) $A_{0}$ is Metzler and $A_{1}, B$ are nonnegative in (3).

Proof. $(b) \Rightarrow(a)$. In this case the system (1) can be presented as follows:

$$
\begin{aligned}
\dot{x}_{1}(t) & =A_{01} x_{1}(t)+A_{02} x_{2}(t)+A_{11} x_{1}(t-\tau)+A_{12} x_{2}(t-\tau)+B_{1} u(t), \\
0 & =A_{03} x_{1}(t)+A_{04} x_{2}(t)+A_{13} x_{1}(t-\tau)+A_{14} x_{2}(t-\tau)+B_{2} u(t)
\end{aligned}
$$

with initial conditions $x_{0}=\left[x_{10}^{T} x_{20}^{T}\right] \in \mathcal{C}_{\tau+}^{n}$. According to [29], [32], $x_{1}(t) \in \mathbb{R}_{+}^{n_{1}}$ for all $t \geq 0$ with $x_{10} \in \mathcal{C}_{\tau+}^{n_{1}}$ provided that $A_{01}$ is Metzler, the matrices $B_{1}, A_{02}, A_{11}$ and $A_{12}$ are nonnegative, and $x_{2}(t) \in \mathbb{R}_{+}^{n_{2}}$ for all $t \geq-\tau$. The latter condition is satisfied for $x_{20} \in \mathcal{C}_{\tau+}^{n_{2}}$ if $A_{04}$ is Hurwitz and Metzler matrix (in this case $A_{04}^{-1} \leq 0$ [34]), the matrices $B_{2}, A_{03}, A_{13}$ and $A_{14}$ are nonnegative, and $x_{1}(t) \in \mathbb{R}_{+}^{n_{1}}$ for all $t \geq-\tau$ :

$$
x_{2}(t)=-A_{04}^{-1}\left[A_{03} x_{1}(t)+A_{13} x_{1}(t-\tau)+A_{14} x_{2}(t-\tau)+B_{2} u(t)\right] .
$$

Summarizing all these restrictions on matrices we obtain that $A_{0}$ should be Metzler with a Hurwitz block $A_{04}$, $A_{1} \geq 0$ and $B \geq 0$ in (2). Therefore, $x_{1}(t) \in \mathbb{R}_{+}$for all $t \geq 0$ if $x_{0} \in \mathcal{C}_{\tau+}^{n}$ and $u(t) \in \mathbb{R}_{+}^{m}, x_{2}(t) \in \mathbb{R}_{+}^{n_{2}}$ for all $t \geq 0$; while $x_{2}(t) \in \mathbb{R}_{+}$for all $t \geq 0$ if $x_{0} \in \mathcal{C}_{\tau+}^{n}$ and $u(t) \in \mathbb{R}_{+}^{m}, x_{1}(t) \in \mathbb{R}_{+}^{n_{2}}$ for all $t \geq 0$. Consequently, the conditions $x_{0} \in \mathcal{C}_{\tau+}^{n}$ and $u(t) \in \mathbb{R}_{+}^{m}$ for all $t \geq 0$ lead to $x(t) \in \mathbb{R}_{+}^{n}$ for all $t \geq 0$.

$(a) \Rightarrow(c)$. If $\operatorname{det}\left(A_{04}\right) \neq 0$ in (2), then without loosing generality we can select (3) with $A_{04}=-I_{n_{2}}$. The claim for the variable $x_{1}$ has been proven in [29], [32] (considering $x_{2}$ as a nonnegative input for all $t \geq-\tau$ ), 
then a proof for the variable $x_{2}$ follows similar arguments (the idea is that if $A_{0}$ is not Metzler for $A_{04}=-I_{n_{2}}$ or $A_{1}, B$ have a negative element, then there are $x_{0} \in \mathcal{C}_{\tau+}^{n}, u(t) \in \mathbb{R}_{+}^{m}$ such that $x(t)$ takes a negative value at some time instant).

Obviously $(c) \Rightarrow(b)$ since (3) is a variant of (2).

\section{Interval arithmetic}

The following lemmas have been proven in [22], [21]. Given a matrix $A \in \mathbb{R}^{m \times n}$, define $A^{+}=\max \{0, A\}$ (elementwise), $A^{-}=A^{+}-A$ (similarly for vectors) and denote the matrix of absolute values of all elements by $|A|=A^{+}+A^{-}$.

Lemma 1. Let $x \in \mathbb{R}^{n}$ be a vector variable, $\underline{x} \leq x \leq \bar{x}$ for some $\underline{x}, \bar{x} \in \mathbb{R}^{n}$, and $A \in \mathbb{R}^{m \times n}$ be a constant matrix, then

$$
A^{+} \underline{x}-A^{-} \bar{x} \leq A x \leq A^{+} \bar{x}-A^{-} \underline{x} .
$$

Lemma 2. Let $\underline{A} \leq A \leq \bar{A}$ for some $\underline{A}, \bar{A}, A \in \mathbb{R}^{n \times n}$ and $\underline{x} \leq x \leq \bar{x}$ for some $\underline{x}, \bar{x}, x \in \mathbb{R}^{n}$, then

$$
\underline{A}^{+} \underline{x}^{+}-\bar{A}^{+} \underline{x}^{-}-\underline{A}^{-} \bar{x}^{+}+\bar{A}^{-} \bar{x}^{-} \leq A x \leq \bar{A}^{+} \bar{x}^{+}-\underline{A}^{+} \bar{x}^{-}-\bar{A}^{-} \underline{x}^{+}+\underline{A}^{-} \underline{x}^{-} .
$$

\section{PROBLEM STATEMENT}

In this work we will consider an extended version of the descriptor time-delay system (1)

$$
\begin{aligned}
E \dot{x}(t) & =A_{0} x(t)+A_{1} x(t-\tau)+B u(t)+d(t), \\
y(t) & =C x(t)+v(t),
\end{aligned}
$$

where as before $\tau>0$ is the delay and $x(t) \in \mathbb{R}^{n}$ is the state (with initial conditions $\left.x_{0} \in \mathcal{C}_{\tau}^{n}\right) ; u(t) \in \mathcal{L}_{\infty}^{m}$ is the control signal; $d(t) \in \mathcal{L}_{\infty}^{n}$ is a disturbance; $y \in \mathbb{R}^{p}$ is the output signal available for measurements; $v(t) \in \mathcal{L}_{\infty}^{p}$ is the measurement noise. The constant matrices $E, A_{0}, A_{1}, B$ and $C$ have appropriate dimensions. The following restrictions are imposed on (5). The case of multiple delays can be incorporated using the results of [27].

Assumption 1. The system (5) is regular with index 1 and presented in the canonical form (2) with $\operatorname{det}\left(A_{04}\right) \neq 0$.

Assumption 2. The state $x(t)$ is a bounded function of time (i.e. $\left.x(t) \in \mathcal{L}_{\infty}^{n}\right)$.

Assumption 3. There is a known constant $V>0$ such that $\left\|v_{i}\right\|_{[0, \infty)} \leq V$ for all $1 \leq i \leq p$, there exist two known functions $\underline{d}, \bar{d}: \mathbb{R}_{+} \rightarrow \mathbb{R}^{n}, \underline{d}(t), \bar{d}(t) \in \mathcal{L}_{\infty}^{n}$ such that $\underline{d}(t) \leq d(t) \leq \bar{d}(t)$ for all $t \geq 0$, and there exist $\underline{x}_{0}, \bar{x}_{0} \in \mathcal{C}_{\tau}^{n}$ such that $\underline{x}_{0} \leq x_{0} \leq \bar{x}_{0}$.

The first assumption claims that the system (5) is regular and it has no impulsive solutions. It is also indicated that (5) is transformed to the canonical representation (that is always possible for a regular systems with index 1). In the second assumption, boundedness of the state is imposed, which is a typical restriction in the estimation theory. In the third assumption it is assumed that the noise is bounded by $V$ and the lower and upper bounds for $d(t)$ are given (an interval of admissible values for $d(t)$ at each instant of time $t \geq 0$ ).

The objective is to design an interval observer for (5), i.e. a dynamical system

$$
\begin{gathered}
\tilde{E} \dot{z}(t)=F\left(z_{t}, y_{t}, u(t), V, \underline{d}(t), \bar{d}(t)\right), z_{0} \in \mathcal{C}_{\tau}^{k}, k>0, \\
\underline{x}(t)=\underline{H}\left(z_{t}, y_{t}, u(t), V, \underline{d}(t), \bar{d}(t)\right), \bar{x}(t)=\bar{H}\left(z_{t}, y_{t}, u(t), V, \underline{d}(t), \bar{d}(t)\right),
\end{gathered}
$$


where $F, \underline{H}, \bar{H}$ are some functionals and $\tilde{E} \in \mathbb{R}^{k \times k}$, such that $\underline{x}(t) \leq x(t) \leq \bar{x}(t)$ for all $t \geq 0$ provided that $\underline{x}_{0} \leq x_{0} \leq \bar{x}_{0}$. In addition, if $\lim _{t \rightarrow+\infty}\|\underline{d}(t)-\bar{d}(t)\|=\lim _{t \rightarrow+\infty}\|v(t)\|=0$, then $\lim _{t \rightarrow+\infty}\|\underline{x}(t)-\bar{x}(t)\|=$ $\lim _{t \rightarrow+\infty}\|x(t)-\bar{x}(t)\|=0$.

\section{MAIN RESUlTS}

First of all note that for any $L_{1}, L_{2} \in \mathbb{R}^{n \times p}$ the system (5) can be rewritten as follows:

$$
\begin{aligned}
E \dot{x}(t)= & A_{0} x(t)+A_{1} x(t-\tau)+B u(t)+d(t)+L_{1}(y(t)-C x(t)-v(t)) \\
& +L_{2}(y(t-\tau)-C x(t-\tau)-v(t-\tau)) \\
= & \left(A_{0}-L_{1} C\right) x(t)+\left(A_{1}-L_{2} C\right) x(t-\tau)+B u(t)+\delta(t)+L_{1} y(t)+L_{2} y(t-\tau),
\end{aligned}
$$

where $\delta(t)=d(t)-L_{1} v(t)-L_{2} v(t-\tau)$, and using the result of Lemma 2, for all $t \geq 0$ :

$$
\begin{gathered}
\underline{\delta}(t) \leq \delta(t) \leq \bar{\delta}(t), \\
\underline{\delta}(t)=\underline{d}(t)-\left(\left|L_{1}\right|+\left|L_{2}\right|\right) 1_{p} V, \bar{\delta}(t)=\bar{d}(t)+\left(\left|L_{1}\right|+\left|L_{2}\right|\right) 1_{p} V .
\end{gathered}
$$

Below, two sets of conditions for $L_{1}$ and $L_{2}$ are considered with two different interval observers. Next, we will skip the requirement of Assumption 2 that the state $x(t)$ is bounded, and in addition to $L_{1}, L_{2}$ we will design a control $u(t)$ dependent on the interval estimates $\underline{x}(t), \bar{x}(t)$ providing the state $x(t)$ stabilization, as it has been done in [21], [35] for ordinary differential equations and time-delay systems (with the delay in the input channel only).

Further, denote

$$
C=\left[\begin{array}{ll}
C_{1} & C_{2}
\end{array}\right], L_{i}=\left[\begin{array}{c}
L_{i 1} \\
L_{i 2}
\end{array}\right], i=1,2,
$$

where $C_{1} \in \mathbb{R}^{p \times n_{1}}, C_{2} \in \mathbb{R}^{p \times n_{2}}, L_{i 1} \in \mathbb{R}^{n_{1} \times p}$ and $L_{i 2} \in \mathbb{R}^{n_{2} \times p}$.

\section{A. The case of a positive system}

Actually we will not assume explicitly in this subsection that $x(t) \in \mathbb{R}_{+}^{n}$ and that (5) is positive, but the conditions imposed on $L_{1}$ and $L_{2}$ imply that the linear part in (6), i.e. the matrices $A_{0}-L_{1} C, A_{1}-L_{2} C$ and $B$, is positive.

Consider the following interval observer:

$$
\begin{aligned}
E \underline{\dot{x}}(t)= & \left(A_{0}-L_{1} C\right) \underline{x}(t)+\left(A_{1}-L_{2} C\right) \underline{x}(t-\tau) \\
& +B u(t)+\underline{\delta}(t)+L_{1} y(t)+L_{2} y(t-\tau), \\
E \dot{\bar{x}}(t)= & \left(A_{0}-L_{1} C\right) \bar{x}(t)+\left(A_{1}-L_{2} C\right) \bar{x}(t-\tau) \\
& +B u(t)+\bar{\delta}(t)+L_{1} y(t)+L_{2} y(t-\tau)
\end{aligned}
$$

with initial conditions $\underline{x}_{0}, \bar{x}_{0}$ given in Assumption 3. As we can conclude from (7), the dynamics of $\underline{x}(t)$ and $\bar{x}(t)$ are independent.

Theorem 1. Let assumptions 1-3 be satisfied, $A_{0}-L_{1} C$ be Metzler with a Hurwitz block $A_{04}-L_{12} C_{2}$, and the matrix $A_{1}-L_{2} C$ be nonnegative. Then in (5), (7), $\underline{x}(t) \leq x(t) \leq \bar{x}(t)$ for all $t \geq 0$. In addition, if $\max _{1 \leq i \leq n_{2}} \mid \lambda_{i}\left(\left[A_{04}-\right.\right.$ $\left.\left.L_{12} C_{2}\right]^{-1}\left[A_{14}-L_{22} C_{2}\right]\right) \mid<1$ and there exist matrices $P \in \mathbb{R}^{n \times n}$ of the form (4) and $U=U^{T} \in \mathbb{R}^{n \times n}$ that for 
some $\gamma>0$ satisfy the LMIs

$$
\begin{gathered}
{\left[\begin{array}{ccc}
\Psi & P^{T} & P^{T}\left(A_{1}-L_{2} C\right) \\
P & -\gamma^{2} I_{n} & 0 \\
\left(A_{1}-L_{2} C\right)^{T} P & 0 & -U
\end{array}\right] \prec 0,} \\
\Psi=P^{T}\left(A_{0}-L_{1} C\right)+\left(A_{0}-L_{1} C\right)^{T} P+U+I_{n}, U \succ 0,
\end{gathered}
$$

then $\underline{x}(t), \bar{x}(t) \in \mathcal{L}_{\infty}^{n}$ for any $\tau>0$ and an $H_{\infty}$ gain from the inputs $\delta-\underline{\delta}$ and $\bar{\delta}-\delta$ to the state estimation errors $x-\underline{x}$ and $\bar{x}-x$, respectively, is less than $\gamma$.

Proof. Since all conditions of Proposition 1 are satisfied, the solutions of interval observer (7) are unique, absolutely continuous and well defined for all $t \geq 0$. Let us introduce into consideration the lower and upper estimation errors $\underline{e}=x-\underline{x}$ and $\bar{e}=\bar{x}-x$, which yield the algebraic-differential equations:

$$
\begin{aligned}
& E \underline{\dot{e}}(t)=\left(A_{0}-L_{1} C\right) \underline{e}(t)+\left(A_{1}-L_{2} C\right) \underline{e}(t-\tau)+\delta(t)-\underline{\delta}(t), \\
& E \dot{\bar{e}}(t)=\left(A_{0}-L_{1} C\right) \bar{e}(t)+\left(A_{1}-L_{2} C\right) \bar{e}(t-\tau)+\bar{\delta}(t)-\delta(t) .
\end{aligned}
$$

Under introduced conditions, these equations are independent and describe positive descriptor linear time-delay systems due to Proposition 3,(b). Since $\delta(t)-\underline{\delta}(t) \geq 0$ and $\bar{\delta}(t)-\delta(t) \geq 0$ by construction for all $t \geq 0$, then $\underline{e}(t) \geq 0, \bar{e}(t) \geq 0$ for all $t \geq 0$ provided that $\underline{e}_{0} \geq 0, \bar{e}_{0} \geq 0$, which imply the required interval estimation of $x(t)$ by $\underline{x}(t), \bar{x}(t)$. Boundedness of $\underline{x}(t), \bar{x}(t)$ follows the result of Proposition 2 under the LMI introduced in the theorem formulation and boundedness of $x(t), v(t), d(t), \underline{d}(t)$ and $\bar{d}(t)$.

Remark 1. Note that the result of Theorem 1 means that if $\delta(t)-\underline{\delta}(t) \rightarrow 0, \bar{\delta}(t)-\delta(t) \rightarrow 0$ with $t \rightarrow+\infty$, then $\underline{x}(t) \rightarrow x(t) \leftarrow \bar{x}(t)$ asymptotically.

Note that the LMIs introduced in the formulation of Theorem 1 imply Hurwitz property of $A_{04}-L_{12} C_{2}$ and they can be rewritten in the form suitable for solution with respect to $L_{1}$ and $L_{2}$.

Corollary 1. If there exist $\mu>0$, matrix variables $W_{1} \in \mathbb{R}^{n \times p}, W_{2} \in \mathbb{R}^{n \times p}, Q \in \mathbb{R}^{n_{2} \times n_{2}}$ having a generic structure, diagonal matrices $S, P \in \mathbb{R}^{n \times n}$ satisfying the following LMIs

$$
\begin{gathered}
{\left[\begin{array}{ccc}
\Psi & P^{T} & P^{T} A_{1}-W_{2} C \\
P & -\gamma^{2} I_{n} & 0 \\
A_{1}^{T} P-C^{T} W_{2}^{T} & 0 & -U
\end{array}\right] \prec 0,} \\
\Psi=P^{T} A_{0}+A_{0}^{T} P-W_{1} C-C^{T} W_{1}^{T}+U+I_{n}, U \succ 0, \\
{\left[\begin{array}{cc}
I_{n_{2}} & A_{14}^{T} P_{3}-C_{2}^{T} W_{22}^{T} \\
P_{3} A_{14}-W_{22} C_{2} & Q
\end{array}\right] \succ 0,} \\
Q+\mu^{2} I_{n_{2}}+\mu\left(P_{3} A_{04}-W_{12} C_{2}\right)+\mu\left(A_{04}^{T} P_{3}-C_{2}^{T} W_{12}^{T}\right) \preceq 0, \\
P^{T} A_{0}-W_{1} C+S \geq 0, P^{T} A_{1}-W_{2} C \geq 0, \\
W_{1}=\left[\begin{array}{l}
W_{11} \\
W_{12}
\end{array}\right], W_{2}=\left[\begin{array}{l}
W_{21} \\
W_{22}
\end{array}\right], \\
P_{1}>0, P_{3}>0, P=\operatorname{diag}\left[P_{1}, P_{3}\right] ; S>0, Q \succ 0,
\end{gathered}
$$

then $L_{1}=P^{-1} W_{1}, L_{2}=P^{-1} W_{2}$ are the required observer gains, which yields all restrictions of Theorem 1. 
Proof. The restriction of Theorem 1

$$
\max _{1 \leq i \leq n_{2}}\left|\lambda_{i}\left(\left[A_{04}-L_{12} C_{2}\right]^{-1}\left[A_{14}-L_{22} C_{2}\right]\right)\right|<1
$$

can be equivalently rewritten as follows $\left[A_{14}-L_{22} C_{2}\right]^{T}\left[A_{04}-L_{12} C_{2}\right]^{-T}\left[A_{04}-L_{12} C_{2}\right]^{-1}\left[A_{14}-L_{22} C_{2}\right] \preceq I_{n_{2}}$. Let $Q \succ 0$ be such that $P_{3} Q^{-1} P_{3} \succeq\left[A_{04}-L_{12} C_{2}\right]^{-T}\left[A_{04}-L_{12} C_{2}\right]^{-1}$, then the inequality

$$
\left[A_{14}-L_{22} C_{2}\right]^{T} P_{3} Q^{-1} P_{3}\left[A_{14}-L_{22} C_{2}\right] \preceq I_{n_{2}} \Leftrightarrow\left(\begin{array}{cc}
I_{n_{2}} & {\left[A_{14}-L_{22} C_{2}\right]^{T} P_{3}} \\
P_{3}\left[A_{14}-L_{22} C_{2}\right] & Q
\end{array}\right) \succeq 0
$$

implies (11). On the other hand, $P_{3} Q^{-1} P_{3} \succeq\left[A_{04}-L_{12} C_{2}\right]^{-T}\left[A_{04}-L_{12} C_{2}\right]^{-1}$ is equivalent to $P_{3}^{-1} Q P_{3}^{-1} \preceq$ $\left[A_{04}-L_{12} C_{2}\right]\left[A_{04}-L_{12} C_{2}\right]^{T}$ or

$$
Q \preceq\left[P_{3} A_{04}-P_{3} L_{12} C_{2}\right]\left[P_{3} A_{04}-P_{3} L_{12} C_{2}\right]^{T} .
$$

Since $(X-Y)(X-Y)^{T} \succeq 0$ for any matrices $X$ and $Y$ of the appropriate dimensions, then $X Y^{T}+Y X^{T} \preceq$ $X X^{T}+Y Y^{T}$ and for $X=-\left[A_{04}-L_{12} C_{2}\right]$ and $Y=\mu I_{n_{2}}, \mu>0$ we obtain

$$
-\mu\left[P_{3} A_{04}-P_{3} L_{12} C_{2}\right]-\mu\left[P_{3} A_{04}-P_{3} L_{12} C_{2}\right]^{T} \preceq\left[P_{3} A_{04}-P_{3} L_{12} C_{2}\right]\left[P_{3} A_{04}-P_{3} L_{12} C_{2}\right]^{T}+\mu^{2} I_{n_{2}} .
$$

In this case, the inequality

$$
Q \preceq-\mu\left[P_{3} A_{04}-P_{3} L_{12} C_{2}\right]-\mu\left[P_{3} A_{04}-P_{3} L_{12} C_{2}\right]-\mu^{2} I
$$

implies (12). Finally, introducing new variables $W_{1}=P L_{1}$ and $W_{2}=P L_{2}$ we obtain the proposed series of LMIs.

In Corollary 1 the matrix $P$ is defined diagonal (i.e. $P_{1} \in \mathbb{R}^{n_{1} \times n_{1}}, P_{3} \in \mathbb{R}^{n_{2} \times n_{2}}$ are diagonal while $P_{2}=0$ that is a restriction with respect to (4), which is however natural for nonnegative systems [34]). The LMIs (8), (9) imply the restriction $\max _{1 \leq i \leq n_{2}}\left|\lambda_{i}\left(\left[A_{04}-L_{12} C_{2}\right]^{-1}\left[A_{14}-L_{22} C_{2}\right]\right)\right|<1$, and (10) ensures that the matrices $A_{0}-L_{1} C$, $A_{1}-L_{2} C$ are Metzler and nonnegative respectively.

\section{B. The case without restriction on $A_{1}-L_{2} C$}

In this part we would like to relax the restrictions introduced in the previous subsection for $L_{1}$ and $L_{2}$. For this purpose consider another interval observer:

$$
\begin{aligned}
E \underline{\dot{x}}(t)= & \left(A_{0}-L_{1} C\right) \underline{x}(t)+\left(A_{1}-L_{2} C\right)^{+} \underline{x}(t-\tau)-\left(A_{1}-L_{2} C\right)^{-} \bar{x}(t-\tau) \\
& +B u(t)+\underline{\delta}(t)+L_{1} y(t)+L_{2} y(t-\tau), \\
E \dot{\bar{x}}(t)= & \left(A_{0}-L_{1} C\right) \bar{x}(t)+\left(A_{1}-L_{2} C\right)^{+} \bar{x}(t-\tau)-\left(A_{1}-L_{2} C\right)^{-} \underline{x}(t-\tau) \\
& +B u(t)+\bar{\delta}(t)+L_{1} y(t)+L_{2} y(t-\tau)
\end{aligned}
$$

with initial conditions $\underline{x}_{0}, \bar{x}_{0}$ from Assumption 3. In this case the dynamics of $\underline{x}, \bar{x}$ are interrelated in (13) and the matrix $A_{1}-L_{2} C$ may have negative elements.

Theorem 2. Let assumptions 1-3 be satisfied and the matrix $A_{0}-L_{1} C$ be Metzler with a Hurwitz block $A_{04}-L_{12} C_{2}$. Then in (5), (13), $\underline{x}(t) \leq x(t) \leq \bar{x}(t)$ for all $t \geq 0$. If $\max _{1 \leq i \leq 2 n_{2}}\left|\lambda_{i}\left(\Lambda_{04}^{-1} \Lambda_{14}\right)\right|<1$ and there exist matrices $P \in \mathbb{R}^{2 n \times 2 n}$,

$$
P=\left[\begin{array}{cc}
P_{1} & 0 \\
P_{2} & P_{3}
\end{array}\right], P_{1}=P_{1}^{T} \succ 0, P_{1} \in \mathbb{R}^{2 n_{1} \times 2 n_{1}}, P_{3} \in \mathbb{R}^{2 n_{2} \times 2 n_{2}}
$$


and $U=U^{T} \in \mathbb{R}^{2 n \times 2 n}$ that for some $\gamma>0$ satisfy the LMIs

$$
\begin{gathered}
{\left[\begin{array}{ccc}
\Psi & P^{T} & P^{T} \Lambda_{1} \\
P & -\gamma^{2} I_{2 n} & 0 \\
\Lambda_{1}^{T} P & 0 & -U
\end{array}\right] \prec 0,} \\
\Psi=P^{T} \Lambda_{0}+\Lambda_{0}^{T} P+U+I_{2 n}, U \succ 0, \Lambda_{i}=\left[\begin{array}{cc}
\Lambda_{i 1} & \Lambda_{i 2} \\
\Lambda_{i 3} & \Lambda_{i 4}
\end{array}\right], i=1,2, \\
\Lambda_{01}=\left[\begin{array}{cc}
A_{01}-L_{11} C_{1} & 0 \\
0 & A_{01}-L_{11} C_{1}
\end{array}\right], \Lambda_{02}=\left[\begin{array}{cc}
A_{02}-L_{11} C_{2} & 0 \\
0 & A_{02}-L_{11} C_{2}
\end{array}\right], \\
\Lambda_{03}=\left[\begin{array}{cc}
A_{03}-L_{12} C_{1} & 0 \\
0 & A_{03}-L_{12} C_{1}
\end{array}\right], \Lambda_{04}=\left[\begin{array}{cc}
A_{04}-L_{12} C_{2} & 0 \\
0 & A_{04}-L_{12} C_{2}
\end{array}\right], \\
\Lambda_{11}=\left[\begin{array}{cc}
\left(A_{11}-L_{21} C_{1}\right)^{+} & \left(A_{11}-L_{21} C_{1}\right)^{-} \\
\left(A_{11}-L_{21} C_{1}\right)^{-} & \left(A_{11}-L_{21} C_{1}\right)^{+}
\end{array}\right], \Lambda_{12}=\left[\begin{array}{cc}
\left(A_{12}-L_{21} C_{2}\right)^{+} & \left(A_{12}-L_{21} C_{2}\right)^{-} \\
\left(A_{12}-L_{21} C_{2}\right)^{-} & \left(A_{12}-L_{21} C_{2}\right)^{+}
\end{array}\right], \\
\left.\begin{array}{lll}
\left(A_{13}-L_{22} C_{1}\right)^{+} & \left(A_{13}-L_{22} C_{1}\right)^{-} \\
\left(A_{13}-L_{22} C_{1}\right)^{-} & \left(A_{13}-L_{22} C_{1}\right)^{+}
\end{array}\right], \Lambda_{14}=\left[\begin{array}{cc}
\left(A_{14}-L_{22} C_{2}\right)^{+} & \left(A_{14}-L_{22} C_{2}\right)^{-} \\
\left(A_{14}-L_{22} C_{2}\right)^{-} & \left(A_{14}-L_{22} C_{2}\right)^{+}
\end{array}\right],
\end{gathered}
$$

then $\underline{x}(t), \bar{x}(t) \in \mathcal{L}_{\infty}^{n}$ for any $\tau>0$ and an $H_{\infty}$ gain from the inputs $\delta-\underline{\delta}, \bar{\delta}-\delta$ to the state estimation errors $x-\underline{x}, \bar{x}-x$ is less than $\gamma$.

Proof. Again, all conditions of Proposition 1 are satisfied, then the solutions of interval observer (13) are unique, absolutely continuous and well defined for all $t \geq 0$. The dynamics of the lower $\underline{e}$ and upper $\bar{e}$ estimation errors admit the algebraic-differential equations:

$$
\begin{aligned}
& E \underline{\dot{e}}(t)=\left(A_{0}-L_{1} C\right) \underline{e}(t)+\left(A_{1}-L_{2} C\right)^{+} \underline{e}(t-\tau)+\left(A_{1}-L_{2} C\right)^{-} \bar{e}(t-\tau)+\delta(t)-\underline{\delta}(t), \\
& E \dot{\bar{e}}(t)=\left(A_{0}-L_{1} C\right) \bar{e}(t)+\left(A_{1}-L_{2} C\right)^{+} \bar{e}(t-\tau)+\left(A_{1}-L_{2} C\right)^{-} \underline{e}(t-\tau)+\bar{\delta}(t)-\delta(t) .
\end{aligned}
$$

Introducing the partition $\underline{e}=\left[\underline{e}_{1}^{\mathrm{T}} \underline{e}_{2}^{\mathrm{T}}\right]^{\mathrm{T}}, \bar{e}=\left[\bar{e}_{1}^{\mathrm{T}} \bar{e}_{2}^{\mathrm{T}}\right]^{\mathrm{T}}$ and similarly for $\delta, \underline{\delta}, \bar{\delta}$ we obtain:

$$
\begin{aligned}
\underline{e}_{1}(t)= & \left(A_{01}-L_{11} C_{1}\right) \underline{e}_{1}(t)+\left(A_{02}-L_{11} C_{2}\right) \underline{e}_{2}(t)+\left(A_{11}-L_{21} C_{1}\right)^{+} \underline{e}_{1}(t-\tau)+\left(A_{12}-L_{21} C_{2}\right)^{+} \underline{e}_{2}(t-\tau) \\
& +\left(A_{11}-L_{21} C_{1}\right)^{-} \bar{e}_{1}(t-\tau)+\left(A_{12}-L_{21} C_{2}\right)^{-} \bar{e}_{2}(t-\tau)+\delta_{1}(t)-\underline{\delta}_{1}(t), \\
0= & \left(A_{03}-L_{12} C_{1}\right) \underline{e}_{1}(t)+\left(A_{04}-L_{12} C_{2}\right) \underline{e}_{2}(t)+\left(A_{13}-L_{22} C_{1}\right)^{+} \underline{e}_{1}(t-\tau)+\left(A_{14}-L_{22} C_{2}\right)^{+} \underline{e}_{2}(t-\tau) \\
& +\left(A_{13}-L_{22} C_{1}\right)^{-} \bar{e}_{1}(t-\tau)+\left(A_{14}-L_{22} C_{2}\right)^{-} \bar{e}_{2}(t-\tau)+\delta_{2}(t)-\underline{\delta}_{2}(t), \\
\dot{\bar{e}}_{1}(t)= & \left(A_{01}-L_{11} C_{1}\right) \bar{e}_{1}(t)+\left(A_{02}-L_{11} C_{2}\right) \bar{e}_{2}(t)+\left(A_{11}-L_{21} C_{1}\right)^{+} \bar{e}_{1}(t-\tau)+\left(A_{12}-L_{21} C_{2}\right)^{+} \bar{e}_{2}(t-\tau) \\
& +\left(A_{11}-L_{21} C_{1}\right)^{-} \underline{e}_{1}(t-\tau)+\left(A_{12}-L_{21} C_{2}\right)^{-} \underline{e}_{2}(t-\tau)+\bar{\delta}_{1}(t)-\delta_{1}(t), \\
0 & \left(A_{03}-L_{12} C_{1}\right) \bar{e}_{1}(t)+\left(A_{04}-L_{12} C_{2}\right) \bar{e}_{2}(t)+\left(A_{13}-L_{22} C_{1}\right)^{+} \bar{e}_{1}(t-\tau)+\left(A_{14}-L_{22} C_{2}\right)^{+} \bar{e}_{2}(t-\tau) \\
& +\left(A_{13}-L_{22} C_{1}\right)^{-} \underline{e}_{1}(t-\tau)+\left(A_{14}-L_{22} C_{2}\right)^{-} \underline{e}_{2}(t-\tau)+\bar{\delta}_{2}(t)-\delta_{2}(t) .
\end{aligned}
$$

Introducing a common error vector $\epsilon=\left[\underline{e}_{1}^{\mathrm{T}} \bar{e}_{1}^{\mathrm{T}} \underline{e}_{2}^{\mathrm{T}} \bar{e}_{2}^{\mathrm{T}}\right]^{\mathrm{T}}$ we can rewrite the dynamics of $\epsilon$ in the descriptor form:

$$
\Sigma \dot{\epsilon}(t)=\Lambda_{0} \epsilon(t)+\Lambda_{1} \epsilon(t-\tau)+\rho(t)
$$


where $\rho(t)=\left[\left(\delta_{1}(t)-\underline{\delta}_{1}(t)\right)^{\mathrm{T}}\left(\bar{\delta}_{1}(t)-\delta_{1}(t)\right)^{\mathrm{T}}\left(\delta_{2}(t)-\underline{\delta}_{2}(t)\right)^{\mathrm{T}}\left(\bar{\delta}_{2}(t)-\delta_{2}(t)\right)^{\mathrm{T}}\right]^{\mathrm{T}} \in \mathbb{R}_{+}^{2 n}$ for all $t \geq 0$,

$$
\Sigma=\left[\begin{array}{cc}
I_{2 n_{1}} & 0 \\
0 & 0
\end{array}\right] \text {. }
$$

It is straightforward to check that for the partition $\epsilon=\left[\epsilon_{1}^{\mathrm{T}} \epsilon_{2}^{\mathrm{T}}\right]^{\mathrm{T}}$ with $\epsilon_{1} \in \mathbb{R}^{2 n_{1}}$ and $\epsilon_{2} \in \mathbb{R}^{2 n_{2}}$ the descriptor time-delay system (15) is in the canonical form (2), and due to Proposition 3 and the structure of $\Lambda_{0}, \Lambda_{1}$ the system (15) is positive (if the matrix $A_{0}-L_{1} C$ is Metzler, then the blocks $A_{01}-L_{11} C_{1}, A_{04}-L_{12} C_{2}$ are Metzler and $A_{02}-L_{11} C_{2}, A_{03}-L_{12} C_{1}$ are nonnegative). Thus $\rho(t) \geq 0$ for all $t \geq 0$, then $\epsilon(t) \geq 0$ for all $t \geq 0$ provided that $\epsilon_{0} \geq 0$, which is equivalent to the required interval estimation of $x(t)$ by $\underline{x}(t), \bar{x}(t)$. Since $\operatorname{det}\left(\Lambda_{04}\right) \neq 0$ if $\operatorname{det}\left(A_{04}-L_{12} C_{2}\right) \neq 0$, then boundedness of $\epsilon(t)$ follows from the result of Proposition 2 under the LMI introduced for the system (15) in the theorem formulation and boundedness of $x(t), \rho(t)$ (the latter is bounded if $v(t), d(t)$, $\underline{d}(t)$ and $\bar{d}(t)$ are so).

Contrarily to Theorem 1, the restriction $A_{1}-L_{2} C \geq 0$ is not imposed in Theorem 2, the price for that is more complicated equations of interval observer (check (13) with respect to (7)) and more sophisticated matrix inequalities. The restrictions of Theorem 2 can also be presented as a series of LMIs as in Corollary 1 for Theorem 1 since the conditions of these theorems are similar (this result is skipped for brevity).

Remark 2. The conditions of Theorem 2 may be relaxed asking existence of a transformation matrix $S \in \mathbb{R}^{n_{1} \times n_{1}}$ such that $S^{-1}\left(A_{0}-L_{1} C\right) S$ is Metzler, the conditions of such a transformation matrix existence can be found in [24] (for Theorem 1 such a relaxation is more complicated since in that case in the new coordinates it is necessary to ensure that $S^{-1}\left(A_{0}-L_{1} C\right) S$ is Metzler and $S^{-1}\left(A_{1}-L_{2} C\right) S$ is nonnegative).

Remark 3. Finally note that a bounded function $d(\cdot)$ can be dependent on measured output $y$ and input $u$ in a nonlinear fashion, which does not change the applicability conditions of theorems 1 and 2 provided that $\underline{d}(t)$ and $\bar{d}(t)$ can be constructed. Thus the proposed interval observers (7) and (13) can be applied for estimation of uncertain nonlinear differential-algebraic systems. The delay $\tau$ can also be considered time-varying and uncertain under a mild modification of the structure of observers (7) and (13) in the way it has been proposed in [20].

\section{Stabilization of descriptor time-delay systems using interval observer (7)}

In this subsection we will try to skip Assumption 2 and, in order to simplify the presentation, we will consider the case of interval observer (7) only (similar results can be obtained for (13), they are omitted for compactness).

In Theorem 1 the gains $L_{1}$ and $L_{2}$ have been used to guarantee the properties of positivity and stability for the dynamics of estimation errors $\underline{e}(t), \bar{e}(t)$. The positivity property has been obtained uniformly in $u(t)$. Thus the control design can be applied in order to ensure boundedness of the observer estimates $\underline{x}(t), \bar{x}(t)$, that in its turn $(\underline{x}(t) \leq x(t) \leq \bar{x}(t)$ for all $t \geq 0)$ will provide boundedness of $x(t)$. An advantage of this approach is that the system (5) is uncertain and the state of that system is not measured, while the observer (7) is a completely known system with the accessible state $\underline{x}(t), \bar{x}(t)$ [21]. A drawback is that the dimension of the state for (7) is $2 n$, while the dimension of the control is $m$, similarly to (5).

It is also necessary to take into account that for stabilization of $\underline{x}(t), \bar{x}(t)$ in (7), the signals $y(t)$ and $y(t-\tau)$ form a kind of functional perturbation, which is globally Lipschitz with respect to $\underline{x}(t), \bar{x}(t)$. Indeed, from the 
relation $\underline{x}(t) \leq x(t) \leq \bar{x}(t)$ we have

$$
\begin{aligned}
\left|x_{i}(t)\right| & \leq \max \left\{\left|\underline{x}_{i}(t)\right|,\left|\bar{x}_{i}(t)\right|\right\} \quad \forall 1 \leq i \leq n, \\
\left|x_{i}(t)\right|^{2} & \leq\left|\underline{x}_{i}(t)\right|^{2}+\left|\bar{x}_{i}(t)\right|^{2} \quad \forall 1 \leq i \leq n, \\
\|x(t)\| & \leq \sqrt{\|\underline{x}(t)\|^{2}+\|\bar{x}(t)\|^{2}} \leq \sqrt{2} \max \{\|\underline{x}(t)\|,\|\bar{x}(t)\|\},
\end{aligned}
$$

then $\|y(t)\| \leq\|C\| \sqrt{2} \max \{\|\underline{x}(t)\|,\|\bar{x}(t)\|\}$.

Take

$$
u(t)=\underline{K}_{1} \underline{x}(t)+\bar{K}_{1} \bar{x}(t)+\underline{K}_{2} \underline{x}(t-\tau)+\bar{K}_{2} \bar{x}(t-\tau)-R_{1} y(t)-R_{2} y(t-\tau)
$$

for some matrices $\underline{K}_{i}, \bar{K}_{i} \in \mathbb{R}^{m \times n}, R_{i} \in \mathbb{R}^{m \times p}$ with $i=1,2$. The idea of introduction of $R_{1}$ and $R_{2}$ is to minimize the influence of $y(t), y(t-\tau)$ on the dynamics of $\underline{x}(t), \bar{x}(t)$ in (7), i.e. to minimize the norm of $F_{i}=L_{i}-B R_{i}$. In a similar way, a term can be introduced in $u(t)$ in order to reduce the influence of $\underline{\delta}(t), \bar{\delta}(t)$.

Theorem 3. Let assumptions 1,3 be satisfied, the matrix $A_{0}-L_{1} C$ be Metzler with a Hurwitz block $A_{04}-L_{12} C_{2}$ and the matrix $A_{1}-L_{2} C$ be nonnegative. Then in (5), (7) with the control (16), $\underline{x}(t) \leq x(t) \leq \bar{x}(t)$ for all $t \geq 0$. If $\max _{1 \leq i \leq n_{2}}\left|\lambda_{i}\left(\Delta_{04}^{-1} \Delta_{14}\right)\right|<1$ and there exist matrices $P \in \mathbb{R}^{2 n \times 2 n}$ of the form (14) and $U=U^{T} \in \mathbb{R}^{2 n \times 2 n}$ that for $\gamma<\left[\| C|| 2 \sqrt{2}\left(\left\|F_{1}\right\|+\left\|F_{2}\right\|\right)\right]^{-1}$ satisfy the matrix inequalities

$$
\begin{aligned}
& {\left[\begin{array}{ccc}
\Psi & P^{T} & P^{T} \Delta_{1} \\
P & -\gamma^{2} I_{2 n} & 0 \\
\Delta_{1}^{T} P & 0 & -U
\end{array}\right] \prec 0,} \\
& \Psi=P^{T} \Delta_{0}+\Delta_{0}^{T} P+U+I_{2 n}, U \succ 0, \Delta_{i}=\left[\begin{array}{cc}
\Delta_{i 1} & \Delta_{i 2} \\
\Delta_{i 3} & \Delta_{i 4}
\end{array}\right], i=1,2, \\
& \Delta_{01}=\left[\begin{array}{cc}
A_{01}-L_{11} C_{1}+B_{1} \underline{K}_{11} & B_{1} \bar{K}_{11} \\
B_{1} \underline{K}_{11} & A_{01}-L_{11} C_{1}+B_{1} \bar{K}_{11}
\end{array}\right], \Delta_{02}=\left[\begin{array}{cc}
A_{02}-L_{11} C_{2}+B_{1} \underline{K}_{12} & B_{1} \bar{K}_{12} \\
B_{1} \underline{K}_{12} & A_{02}-L_{11} C_{2}+B_{1} \bar{K}_{12}
\end{array}\right], \\
& \Delta_{03}=\left[\begin{array}{cc}
A_{03}-L_{12} C_{1}+B_{2} \underline{K}_{11} & B_{2} \bar{K}_{11} \\
B_{2} \underline{K}_{11} & A_{03}-L_{12} C_{1}+B_{2} \bar{K}_{11}
\end{array}\right], \Delta_{04}=\left[\begin{array}{cc}
A_{04}-L_{12} C_{2}+B_{2} \underline{K}_{12} & B_{2} \bar{K}_{12} \\
B_{2} \underline{K}_{12} & A_{04}-L_{12} C_{2}+B_{2} \bar{K}_{12}
\end{array}\right] \text {, } \\
& \Delta_{11}=\left[\begin{array}{cc}
A_{11}-L_{21} C_{1}+B_{1} \underline{K}_{21} & B_{1} \bar{K}_{21} \\
B_{1} \underline{K}_{21} & A_{11}-L_{21} C_{1}+B_{1} \bar{K}_{21}
\end{array}\right], \Delta_{12}=\left[\begin{array}{cc}
A_{12}-L_{21} C_{2}+B_{1} \underline{K}_{22} & B_{1} \bar{K}_{22} \\
B_{1} \underline{K}_{22} & A_{12}-L_{21} C_{2}+B_{1} \bar{K}_{22}
\end{array}\right], \\
& \Delta_{13}=\left[\begin{array}{cc}
A_{13}-L_{22} C_{1}+B_{2} \underline{K}_{21} & B_{2} \bar{K}_{21} \\
B_{2} \underline{K}_{21} & A_{13}-L_{22} C_{1}+B_{2} \bar{K}_{21}
\end{array}\right], \Delta_{14}=\left[\begin{array}{cc}
A_{14}-L_{22} C_{2}+B_{2} \underline{K}_{22} & B_{2} \bar{K}_{22} \\
B_{2} \underline{K}_{22} & A_{14}-L_{22} C_{2}+B_{2} \bar{K}_{22}
\end{array}\right] \text {, }
\end{aligned}
$$

then $x(t), \underline{x}(t), \bar{x}(t) \in \mathcal{L}_{\infty}^{n}$ for any $\tau>0$ and an $H_{\infty}$ gain from the inputs $\underline{\delta}, \bar{\delta}$ to the states $\underline{x}, \bar{x}$ is less than $\gamma$ and to the state $x$ is less than $\sqrt{2} \gamma$.

Proof. Substitution of the control (16) into equations of the interval observer (7) gives:

$$
\begin{aligned}
E \underline{\dot{x}}(t)= & \left(A_{0}-L_{1} C+B \underline{K}_{1}\right) \underline{x}(t)+\left(A_{1}-L_{2} C+B \underline{K}_{2}\right) \underline{x}(t-\tau) \\
& +B\left[\bar{K}_{1} \bar{x}(t)+\bar{K}_{2} \bar{x}(t-\tau)\right]+\underline{\delta}(t)+F_{1} y(t)+F_{2} y(t-\tau), \\
E \dot{\bar{x}}(t)= & \left(A_{0}-L_{1} C+B \bar{K}_{1}\right) \bar{x}(t)+\left(A_{1}-L_{2} C+B \bar{K}_{2}\right) \bar{x}(t-\tau) \\
& +B\left[\underline{K}_{1} \underline{x}(t)+\underline{K}_{2} \underline{x}(t-\tau)\right]+\bar{\delta}(t)+F_{1} y(t)+F_{2} y(t-\tau) .
\end{aligned}
$$

Introducing partition of matrices $\underline{K}_{i}, \bar{K}_{i}, F_{i}$ on the corresponding blocks with dimensions $n_{1}$ and $n_{2}$, and denoting the common state vector by $\xi=\left[\underline{x}_{1}^{\mathrm{T}} \bar{x}_{1}^{\mathrm{T}} \underline{x}_{2}^{\mathrm{T}} \bar{x}_{2}^{\mathrm{T}}\right]$, we can rewrite the dynamics of $\underline{x}(t), \bar{x}(t)$ in the descriptor canonical 
form:

$$
\Sigma \dot{\xi}(t)=\Delta_{0} \xi(t)+\Delta_{1} \xi(t-\tau)+\mu\left(t, y_{t}\right),
$$

where $\mu\left(t, y_{t}\right)=\left[\left(\underline{\delta}_{1}(t)+F_{11} y(t)+F_{21} y(t-\tau)\right)^{\mathrm{T}}\left(\bar{\delta}_{1}(t)+F_{11} y(t)+F_{21} y(t-\tau)\right)^{\mathrm{T}}\left(\underline{\delta}_{2}(t)+F_{12} y(t)+F_{22} y(t-\right.\right.$ $\left.\tau))^{\mathrm{T}}\left(\bar{\delta}_{2}(t)+F_{12} y(t)+F_{22} y(t-\tau)\right)^{\mathrm{T}}\right]^{\mathrm{T}} \in \mathbb{R}^{2 n}$,

$$
\Sigma=\left[\begin{array}{cc}
I_{2 n_{1}} & 0 \\
0 & 0
\end{array}\right] .
$$

Since the signals $\underline{\delta}(t), \bar{\delta}(t)$ are bounded and $\|y(t)\| \leq \sqrt{2}\|C\|\|\xi(t)\|$, then the input $\mu$ can be considered as a globally Lipschitz nonlinearity with the gain $\gamma_{\mu}=\| C|| 2 \sqrt{2}\left(\left\|F_{1}\right\|+\left\|F_{2}\right\|\right)$. If it is possible to ensure stability of the system (17) with an $H_{\infty}$ gain $\gamma$ from the input $\mu$ to the state $\xi$ such that $\gamma<\gamma_{\mu}^{-1}$, then by the standard small-gain arguments the system is asymptotically stable if $\underline{\delta}(t)=\bar{\delta}(t)=0$ for all $t \geq 0$, and it has an $H_{\infty}$ gain $\gamma$ from the input $\left[\underline{\delta}(t)^{\mathrm{T}} \bar{\delta}(t)^{\mathrm{T}}\right]^{\mathrm{T}}$ to the state $\xi(t)$ [36]. Following Proposition 2, the conditions of these stability properties are fixed by the matrix inequalities introduced in the theorem formulation. Similar properties for $x(t)$ follow.

In order to calculate $L_{i}, \underline{K}_{i}, \bar{K}_{i}, i=1,2$ the conditions of Theorem 3 can be decoupled. First, asking for $L_{i}$ such that $A_{0}-L_{1} C$ is Metzler and Hurwitz and $A_{1}-L_{2} C$ is nonnegative and Schur stable. Second, looking for $\underline{K}_{i}, \bar{K}_{i}$ such that the LMI in the formulation of Theorem 3 is satisfied (applying a technique similar to Corollary 1).

\section{Simulations}

In this section, three examples of numerical experiments are given for the interval observers (7) and (13). Note that on a finite time interval used for simulation the class of inputs $L_{\infty}$ is included in $L_{2}$ (assuming that inputs $d(t)$ and $v(t)$ equal zero after the end of simulation).

\section{A. Leontief delayed model}

In economics, the Leontief model is a quantitative technique representing the interdependency between production of different commodities [37], [38], [8]. Using designations of (5), $x_{i}$ represents production of $i$ th commodity, $A_{0}-I$ represents the rate of production of commodities, $E$ corresponds to stock placement of commodities, $A_{1}$ may give the influence of the past production, the input $B u(t)$ corresponds to the known supply rate and the disturbance $d(t)$ presents the supply uncertainty, $y$ corresponds to the productions of commodities available for evaluation, $v(t)$ is the error of such an evaluation. It is required to reconstruct the vector of production of all commodities $x$. For simulations we will use in this section

$$
\begin{gathered}
A_{0}=\frac{1}{10}\left[\begin{array}{ccccc}
-9 & 1 & 3 & 2 & 1 \\
4 & -8 & 2 & 3 & 2 \\
1 & 1 & -10 & 1 & 4 \\
0 & 1 & 2 & -10 & 0 \\
1 & 1 & 0 & 0 & -10
\end{array}\right], A_{1}=\frac{1}{10}\left[\begin{array}{ccccc}
-1 & 0 & 0 & 0 & 1 \\
0 & 1 & 0 & 1 & 0 \\
-1 & 0 & 2 & 2 & 2 \\
0 & 0 & 2 & 1 & 0 \\
-1 & 1 & 0 & 0 & 2
\end{array}\right], \\
E=\left[\begin{array}{cc}
I_{3} & 0 \\
0 & 0
\end{array}\right], B=1_{5}, C=[1,0,0,0,0]^{\mathrm{T}}, \\
d(t)=\delta\left[\begin{array}{c}
\sin \left(5 x_{1}+t\right) \\
\cos (t) \\
\sin \left(2 x_{3}\right) \\
\sin \left(3 x_{2}\right) \\
\cos (4 t)
\end{array}\right], u(t)=1+0.25 \sin \left(\frac{t}{2}\right), v(t)=V \sin (25 t), \delta=0.5, V=0.2, \tau=0.1,
\end{gathered}
$$



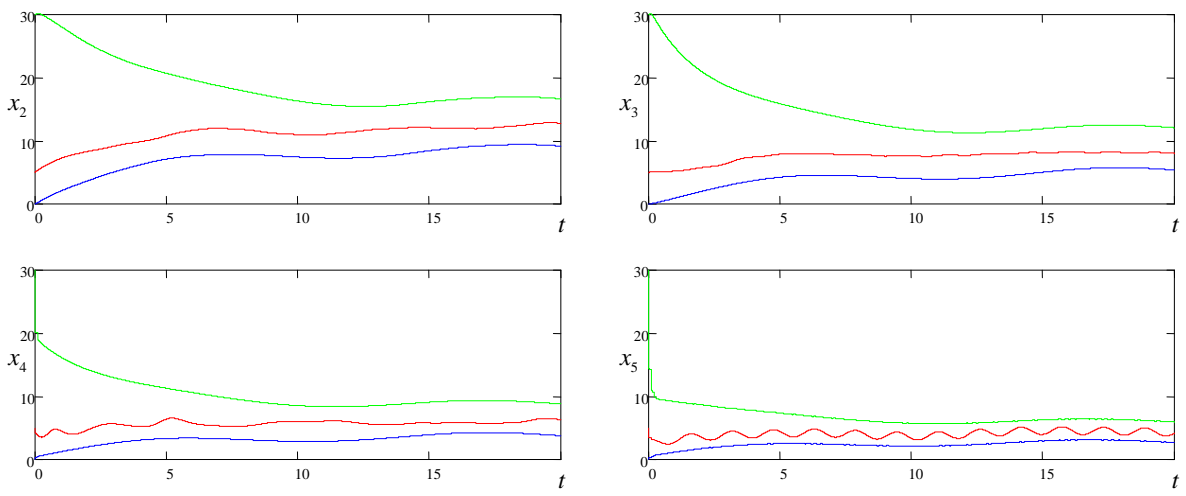

Figure 1. The results of simulation for Leontief model

where $d$ is unknown (the model uncertainty) and only the value of $\delta$ is available. The system is nonlinear since $d$ depends on $x$. Take

$$
L_{1}=\left[\begin{array}{c}
17.39 \\
0.37 \\
0.074 \\
-0.02 \\
0.078
\end{array}\right], L_{2}=\left[\begin{array}{c}
-1.62 \\
-0.027 \\
-0.125 \\
-0.026 \\
-0.12
\end{array}\right],
$$

then $\underline{d}=-\delta 1_{5}=-\bar{d}$ and the LMI of Theorem 1 is satisfied for $\gamma=3 \sqrt{3}, P=\operatorname{diag}[1.06,4.05,4.59,5.86,10.1]$ and

$$
U=\left[\begin{array}{ccccc}
10.81 & 0.04 & -0.02 & -0.1 & 0.21 \\
0.04 & 2.62 & -0.81 & -0.88 & -0.77 \\
-0.02 & -0.81 & 3.66 & -0.78 & -0.78 \\
-0.1 & -0.88 & -0.78 & 4.74 & 0.05 \\
0.21 & -0.77 & -0.78 & 0.05 & 6.91
\end{array}\right]
$$

All conditions of this theorem are valid and the results of interval observer (7) application are given in Fig. 1, for the unmeasured commodities $x_{2}-x_{5}$ (the initial uncertainty for each $x_{i}$ has been assumed in the interval $[0,30]$ ). In each figure red lines correspond to the coordinates $x_{i}$, blue and green lines represent $\underline{x}_{i}$ and $\bar{x}_{i}$ respectively. Due to presence of the system uncertainty $\left(\bar{\delta}(t)-\underline{\delta}(t) \neq 0\right.$ in (6)) the estimates $\underline{x}_{i}, \bar{x}_{i}$ do not converge to the real states $x_{i}$, but they provide an interval estimate of the state with accuracy proportional to $\gamma$.

\section{B. Academic example: estimation}

Let us consider system (5) with

$$
\begin{gathered}
A_{0}=\left[\begin{array}{cccc}
-1 & 0.5 & 0.5 & 0 \\
-1 & -1 & 0 & 0.1 \\
0 & 0.4 & -1 & 0 \\
-0.5 & 0.3 & 0 & -1
\end{array}\right], A_{1}=\left[\begin{array}{cccc}
0 & -0.1 & 0.1 & 0 \\
-0.5 & 0.5 & 0.2 & 0 \\
0.2 & 0 & 0 & -0.1 \\
0.1 & -0.3 & 0 & 0
\end{array}\right], \\
E=\left[\begin{array}{cc}
I_{2} & 0 \\
0 & 0
\end{array}\right], B=\left[\begin{array}{c}
0 \\
1 \\
1 \\
-2
\end{array}\right], C=\left[\begin{array}{c}
1 \\
0 \\
0 \\
0
\end{array}\right], \\
d(t)=\left[\begin{array}{c}
\sin \left(\frac{x_{1}}{3}\right)+\delta \sin \left(x_{2}\right) \\
\sin \left(\frac{x_{1}}{3}\right)-\delta \cos (2 t) \\
\sin \left(\frac{x_{1}}{2}\right)+\delta \sin (t) \\
\sin \left(\frac{x_{1}}{2}\right)+\delta \cos \left(x_{1} t\right)
\end{array}\right], u(t)=\frac{1}{2} \sin \left(\frac{t}{2}\right), v(t)=V \sin (5 t), \delta=0.25, V=0.1, \tau=0.5,
\end{gathered}
$$


where the part of $d$ proportional to $\delta$ is unknown (the model uncertainty). The system is nonlinear since $d$ depends on $x$, however the known part of $d$ depends on $x_{1}(t)=y(t)-v(t)$, then we can design $\underline{d}(t)$ and $\bar{d}(t)$ dependent on the measured signal $y, \delta$ and $V$ only:

$$
\begin{aligned}
& \underline{d}(t)=\left[\begin{array}{l}
\underline{\sin }\left(\frac{y(t)}{3}, \frac{V}{3}\right)-\delta \\
\underline{\sin }\left(\frac{y(t)}{3}, \frac{V}{3}\right)-\delta \\
\underline{\sin \left(\frac{y(t)}{2}, \frac{V}{2}\right)-\delta} \\
\underline{\sin }\left(\frac{y(t)}{2}, \frac{V}{2}\right)-\delta
\end{array}\right], \bar{d}(t)=\left[\begin{array}{l}
\overline{\sin }\left(\frac{y(t)}{3}, \frac{V}{3}\right)+\delta \\
\overline{\sin }\left(\frac{y(t)}{3}, \frac{V}{3}\right)+\delta \\
\overline{\sin }\left(\frac{y(t)}{2}, \frac{V}{2}\right)+\delta \\
\overline{\sin }\left(\frac{y(t)}{2}, \frac{V}{2}\right)+\delta
\end{array}\right], \\
& \underline{\sin }(y, V)=\sin (y)\left\{\begin{array}{ll}
\underline{c}(V) & \text { if } \sin (y) \geq 0 \\
\bar{c}(V) & \text { if } \sin (y)<0
\end{array}-\cos (y)\left\{\begin{array}{ll}
\bar{s}(V) & \text { if } \cos (y) \geq 0 \\
\underline{s}(V) & \text { if } \cos (y)<0
\end{array}\right. \text {, }\right. \\
& \overline{\sin }(y, V)=\sin (y)\left\{\begin{array}{ll}
\bar{c}(V) & \text { if } \sin (y) \geq 0 \\
\underline{c}(V) & \text { if } \sin (y)<0
\end{array}-\cos (y)\left\{\begin{array}{ll}
\underline{s}(V) & \text { if } \cos (y) \geq 0 \\
\bar{s}(V) & \text { if } \cos (y)<0
\end{array},\right.\right. \\
& \underline{c}(V)=\min _{-V \leq v \leq V} \cos (v)=\left\{\begin{array}{ll}
-1 & \text { if } V \geq \pi \\
\cos (V) & \text { if } V<\pi
\end{array}, \bar{c}(V)=\max _{-V \leq v \leq V} \cos (v)=1,\right. \\
& \underline{s}(V)=\min _{-V \leq v \leq V} \sin (v)=\left\{\begin{array}{ll}
-1 & \text { if } V \geq \frac{\pi}{2} \\
-\sin (V) & \text { if } V<\frac{\pi}{2}
\end{array}, \bar{s}(V)=\max _{-V \leq v \leq V} \sin (v)=\left\{\begin{array}{ll}
1 & \text { if } V \geq \frac{\pi}{2} \\
\sin (V) & \text { if } V<\frac{\pi}{2}
\end{array} .\right.\right.
\end{aligned}
$$

Take

$$
L_{1}=\left[\begin{array}{c}
2 \\
-1 \\
0 \\
-0.5
\end{array}\right], L_{2}=\left[\begin{array}{c}
0 \\
-0.5 \\
0.2 \\
0.1
\end{array}\right]
$$

then the matrix $A_{0}-L_{1} C$ is Metzler, but the matrix $A_{1}-L_{2} C$ contains negative elements, which cannot be compensated by the term $L_{2} C$. Thus Theorem 1 cannot be applied here, however the LMI of Theorem 2 is satisfied for $\gamma=4$,

$$
\begin{aligned}
& P=\left[\begin{array}{cccccccc}
46.17 & -12.62 & 2.47 & -2.66 & 0 & 0 & 0 & 0 \\
-12.62 & 10.44 & -2.67 & -0.52 & 0 & 0 & 0 & 0 \\
2.47 & -2.67 & 46.29 & -12.65 & 0 & 0 & 0 & 0 \\
-2.66 & -0.52 & -12.65 & 10.44 & 0 & 0 & 0 & 0 \\
-2.18 & -5.2 & -0.76 & -0.01 & 14.43 & -0.33 & -0.34 & -0.03 \\
-5.2 & -1.86 & -0.04 & -2.52 & -0.33 & 11.97 & -0.02 & -0.86 \\
-0.76 & -0.04 & -1.93 & -5.29 & -0.34 & -0.02 & 14.45 & -0.3 \\
-0.01 & -2.52 & -5.29 & -1.85 & -0.03 & -0.86 & -0.3 & 11.97
\end{array}\right] \\
& U=\left[\begin{array}{cccccccc}
62.68 & -8.46 & -2.32 & -2.68 & -18.07 & -0.02 & 0.36 & 3.7 \\
-8.46 & 8.05 & -2.63 & 1.17 & 0.79 & -2.61 & 0.65 & -2.54 \\
-2.32 & -2.63 & 62.8 & -8.61 & 0.32 & 3.64 & -18.15 & -0.29 \\
-2.68 & 1.17 & -8.61 & 8.04 & 0.66 & -2.53 & 0.8 & -2.62 \\
-18.07 & 0.79 & 0.32 & 0.66 & 10.5 & -0.47 & -0.3 & -0.46 \\
-0.02 & -2.61 & 3.64 & -2.53 & -0.47 & 7.46 & -0.45 & -0.49 \\
0.36 & 0.65 & -18.15 & 0.8 & -0.3 & -0.45 & 10.5 & -0.46 \\
3.7 & -2.54 & -0.29 & -2.62 & -0.46 & -0.49 & -0.46 & 7.48
\end{array}\right]
\end{aligned}
$$

All conditions of this theorem are valid and the results of interval observer (13) application are given in Fig. 2, for the unmeasured coordinates $x_{2}-x_{4}$ (red lines correspond to the coordinates $x_{i}$, blue and green lines represent $\underline{x}_{i}$ and $\bar{x}_{i}$ respectively).

\section{Academic example: stabilization}

Consider system (5) with

$$
A_{0}=\left[\begin{array}{ccc}
1 & 1 & 2 \\
0.1 & -0.5 & 0 \\
0 & 0.1 & -1
\end{array}\right], A_{1}=\left[\begin{array}{ccc}
-0.1 & 0 & 0 \\
0.2 & 0.1 & 0.1 \\
0.1 & 0 & -0.01
\end{array}\right], E=\left[\begin{array}{cc}
I_{2} & 0 \\
0 & 0
\end{array}\right], B=\left[\begin{array}{l}
1 \\
0 \\
0
\end{array}\right], C=\left[\begin{array}{l}
1 \\
0 \\
1
\end{array}\right]^{\mathrm{T}},
$$



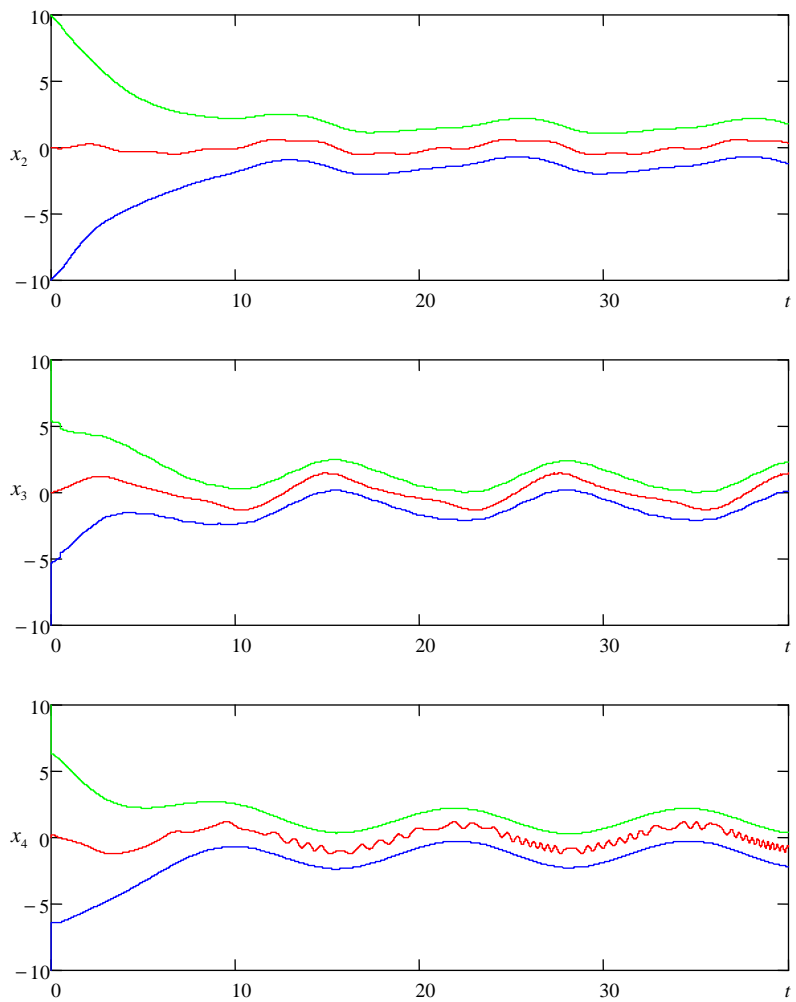

Figure 2. The results of simulation of the interval observer (13)

$$
d(t)=\delta\left[\begin{array}{c}
\sin (0.3 t) \\
\sin (t) \\
\sin (2 t)
\end{array}\right], v(t)=V \sin (15 t), \delta=0.5, V=0.1, \tau=0.1
$$

where it assumed that the information available about disturbance $d$ is $\underline{d}=-\delta 1_{3}=-\bar{d}$. It is straightforward to check that the matrices $A_{0}$ and $A_{0}+A_{1}$ have positive eigenvalues and the system is unstable for $u(t)=0$. Take

$$
L_{1}=\left[\begin{array}{l}
2 \\
0 \\
0
\end{array}\right], L_{2}=\left[\begin{array}{c}
-0.1 \\
0 \\
-0.01
\end{array}\right]
$$

then the matrix $A_{0}-L_{1} C$ is Metzler and the matrix $A_{1}-L_{2} C$ is nonnegative. Assign

$$
\begin{gathered}
\underline{K}_{1}=-\left[\begin{array}{lll}
1 & 1 & 2
\end{array}\right], \bar{K}_{1}=-\left[\begin{array}{lll}
2 & 1 & 1
\end{array}\right], \\
\underline{K}_{2}=-\left[\begin{array}{lll}
0.1 & 1 & 2
\end{array}\right], \bar{K}_{2}=-\left[\begin{array}{lll}
1 & 1 & 4
\end{array}\right], \\
R_{1}=2, R_{2}=-0.1
\end{gathered}
$$

then the conditions of Theorem 3 are satisfied for $\gamma=22$,

$$
P=\left[\begin{array}{cccccc}
47.88 & -32.07 & -28.20 & 42.74 & 0 & 0 \\
-32.07 & 125.38 & 41.64 & 51.09 & 0 & 0 \\
-28.20 & 41.64 & 54.83 & -23.89 & 0 & 0 \\
42.74 & 51.09 & -23.89 & 207.82 & 0 & 0 \\
-5.93 & -62.08 & -58.61 & 0.99 & 200.22 & 79.34 \\
5.34 & -5.04 & -11.82 & -69.85 & 79.34 & 520.68
\end{array}\right], U=\left[\begin{array}{cccccccc}
56.05 & -32.83 & -1.72 & 41.11 & 28.37 & 8.86 \\
-32.83 & 76.74 & 43.24 & -4.33 & -33.84 & 31.10 \\
-1.72 & 43.24 & 79.10 & -16.80 & 5.08 & 44.82 \\
41.11 & -4.33 & -16.80 & 93.19 & 28.13 & -17.38 \\
28.37 & -33.84 & 5.08 & 28.13 & 173.58 & 65.61 \\
8.86 & 31.10 & 44.82 & -17.38 & 65.61 & 273.49
\end{array}\right] .
$$




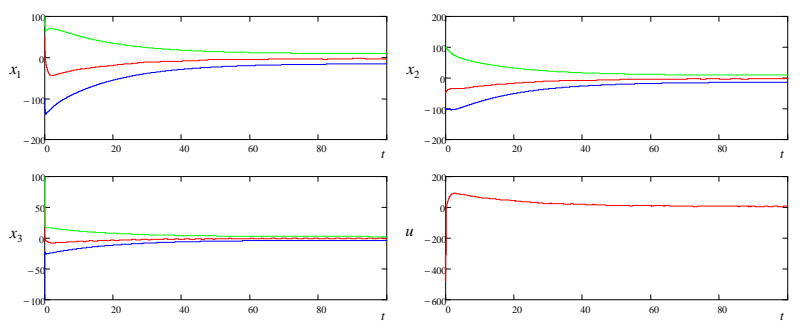

Figure 3. The results of stabilization using the interval observer (7)

The results of interval observer (7) application are given in Fig. 3 together with the control input (16), again red lines correspond to the coordinates $x_{i}$, blue and green lines represent $\underline{x}_{i}$ and $\bar{x}_{i}$ respectively. Stabilization to a zone is achieved only due to presence of measurement noise $(V \neq 0)$ and uncertain input $d$.

\section{CONCLUSION}

For a class of regular descriptor linear systems of index 1 with delays two interval observers are proposed. Two sets of conditions are developed for these observers guaranteeing an interval estimation in the presence of any control input. Next, a control input is designed based on interval estimates providing interval estimation and stabilization simultaneously. Efficiency of the proposed approach is demonstrated in simulation.

As directions of future works, development of computationally less restrictive and simpler LMIs than given in Corollary 1, delay-dependent stability conditions, analysis of time-varying delays or relaxation of Assumption 1, can be considered.

\section{REFERENCES}

[1] G. Besançon, ed., Nonlinear Observers and Applications, vol. 363 of Lecture Notes in Control and Information Sciences. Springer, 2007.

[2] T. Meurer, K. Graichen, and E.-D. Gilles, eds., Control and Observer Design for Nonlinear Finite and Infinite Dimensional Systems, vol. 322 of Lecture Notes in Control and Information Sciences. Springer, 2005.

[3] T. Fossen and H. Nijmeijer, New Directions in Nonlinear Observer Design. Springer, 1999.

[4] J. Gouzé, A. Rapaport, and M. Hadj-Sadok, "Interval observers for uncertain biological systems," Ecological Modelling, vol. 133, pp. 46-56, 2000.

[5] L. Jaulin, "Nonlinear bounded-error state estimation of continuous time systems," Automatica, vol. 38, no. 2, pp. 1079-1082, 2002.

[6] M. Kieffer and E. Walter, "Guaranteed nonlinear state estimator for cooperative systems," Numerical Algorithms, vol. 37, pp. 187-198, 2004.

[7] G.-R. Duan, Analysis and Design of Descriptor Linear Systems, vol. 23 of Advances in Mechanics and Mathematics. Springer, 2010.

[8] M. Silva and T. de Lima, "Looking for nonnegative solutions of a Leontief dynamic model," Linear Algebra, vol. 364, pp. 281-316, 2003.

[9] S. Campbell, Singular Systems of Differential Equations. London: Pitman, 1980.

[10] R. Schüpphaus and P. Müller, "Control analysis and synthesis of linear mechanical descriptor systems," in Advanced Multibody System Dynamics (W. Schiehlen, ed.), vol. 20 of Solid Mechanics and Its Applications, pp. 463-468, Springer Netherlands, 1993.

[11] P. Losse, V. Mehrmann, L. Poppe, and T. Reis, "The modified optimal $H_{\infty}$ control problem for descriptor systems," SIAM Journal Control and Optimization, vol. 47, pp. 2795-2811, 2008.

[12] V. Kolmanovskii and A. Myshkis, Introduction to the Theory and Applications of Functional Differential Equations. Dordrecht: Kluwer Academic Publishers, 1999.

[13] R. Sipahi, S.-I. Niculescu, C. Abdallah, W. Michiels, and K. Gu, "Stability and stabilization of systems with time delay limitations and opportunities," IEEE Control Systems Magazine, vol. 31, no. 1, pp. 38-65, 2011.

[14] J.-P. Richard, "Time delay systems: an overview of some recent advances and open problems," Automatica, vol. 39, no. 10, pp. 16671694, 2003.

[15] E. Fridman, "Descriptor discretized Lyapunov functional method: Analysis and design," IEEE Transactions on Automatic Control, vol. 51, no. 5, pp. 890-897, 2006. 
[16] C. Briat, O. Sename, and J.-F. Lafay, "Design of LPV observers for LPV time-delay systems: an algebraic approach,” Int. J. Control, vol. 84, no. 9, pp. 1533-1542, 2011.

[17] M. Darouach, "Linear functional observers for systems with delays in state variables," IEEE Transactions on Automatic Control, vol. 46, no. 3, pp. 491-496, 2001

[18] G. Zheng, J.-P. Barbot, D. Boutat, T. Floquet, and J.-P. Richard, "On observation of time-delay systems with unknown inputs," IEEE Trans. Automatic Control, vol. 56, no. 8, pp. 1973-1978, 2011.

[19] F. Mazenc, S. Niculescu, and O. Bernard, "Exponentially stable interval observers for linear systems with delay," SIAM J. Control Optim., vol. 50, no. 1, pp. 286-305, 2012.

[20] D. Efimov, W. Perruquetti, and J.-P. Richard, “Interval estimation for uncertain systems with time-varying delays," International Journal of Control, vol. 86, no. 10, pp. 1777-1787, 2013.

[21] D. Efimov, T. Raïssi, and A. Zolghadri, "Control of nonlinear and LPV systems: interval observer-based framework," IEEE Trans. Automatic Control, vol. 58, no. 3, pp. 773-782, 2013.

[22] D. Efimov, L. Fridman, T. Raïssi, A. Zolghadri, and R. Seydou, "Interval estimation for LPV systems applying high order sliding mode techniques," Automatica, vol. 48, pp. 2365-2371, 2012.

[23] M. Moisan, O. Bernard, and J. Gouzé, "Near optimal interval observers bundle for uncertain bio-reactors," Automatica, vol. 45, no. 1, pp. 291-295, 2009.

[24] T. Raïssi, D. Efimov, and A. Zolghadri, "Interval state estimation for a class of nonlinear systems," IEEE Trans. Automatic Control, vol. 57, no. 1, pp. 260-265, 2012.

[25] F. Mazenc and O. Bernard, "ISS interval observers for nonlinear systems transformed into triangular systems," International Journal of Robust and Nonlinear Control, vol. 24, no. 7, pp. 1241-1261, 2014.

[26] F. Mazenc, T. Dinh, and S. Niculescu, "Robust interval observers and stabilization design for discrete-time systems with input and output," Automatica, vol. 49, no. 11, pp. 3490-3497, 2013.

[27] E. Fridman and U. Shaked, " $H_{\infty}$ control of linear state-delay descriptor systems: an LMI approach," Linear Algebra and its Applications, vol. 351-352, pp. 271-302, 2002.

[28] E. Virnik, "Stability analysis of positive descriptor systems," Linear Algebra Appl., vol. 429, pp. 2640-2659, 2008.

[29] M. Dambrine and J.-P. Richard, "Stability analysis of time-delay systems," Dynamic Systems and Applications, vol. 2, pp. 405-414, 1993.

[30] H. Smith, Monotone Dynamical Systems: An Introduction to the Theory of Competitive and Cooperative Systems, vol. 41 of Surveys and Monographs. Providence: AMS, 1995.

[31] L. Farina and S. Rinaldi, Positive Linear Systems: Theory and Applications. New York: Wiley, 2000.

[32] W. Haddad and V. Chellaboina, "Stability theory for nonnegative and compartmental dynamical systems with time delay," Syst. Control Letters, vol. 51, pp. 355-361, 2004.

[33] E. Virnik, Analysis of positive descriptor systems. PhD thesis, TU Berlin, Berlin, 2008.

[34] A. Berman and R. Plemmons, Nonnegative matrices in the mathematical sciences. Philadelphia: SIAM Press, 1994.

[35] A. Polyakov, D. Efimov, W. Perruquetti, and J.-P. Richard, "Output stabilization of time-varying input delay systems using interval observation technique,” Automatica, vol. 49, no. 11, pp. 3402-3410, 2013.

[36] A. R. Teel, "Connections between Razumikhin-type theorems and the ISS nonlinear small gain theorem," IEEE Trans. Automat. Control, vol. 43, no. 7, pp. 960-964, 1998 .

[37] W. G. Vogt, M. Mickle, and H. Aldermeshian, “A dynamic Leontief model for a productive system," Proceedings of the IEEE, vol. 63, pp. 438-443, March 1975.

[38] D. G. Luenberger and A. Arbel, "Singular dynamic Leontief systems," Econometrica, vol. 45, pp. 991-995, May 1977. 